\title{
Considering culture in Aboriginal care
}

$\mathrm{C}$ anadian health care providers need to focus more on offering "culturally competent care" and creating "culturally safe" environments for Aboriginal peoples, who tend to avoid seeking medical care because of factors such as negative stereotypes and lingering racism, according to a new report by the Health Council of Canada.

"Many of our Aboriginal peoples are not coming and using our mainstream health services, mainly because they feel that they are not being respected for who they are and for their legitimate health needs," says John Abbott, the council's chief executive officer. "There still is a lot of racism within the communities, and a lot of our health care professionals are not really seeing the Aboriginal people for who they really are."

About half of Aboriginal Canadians live in cities, and efforts to increase cultural competency and safety in urban health centres have been shown to build trust between health care providers and Aboriginal people, lead to an increase in use of health services and even increased morale among health providers, states Empathy, dignity, and respect: Creating cultural safety for Aboriginal people in urban health care (www.healthcouncil canada.ca/tree/Aboriginal_Report_EN _web_final.pdf).

Cultural competency is about "creating a health care environment that is free of racism and stereotypes, where Aboriginal people are treated with empathy, dignity and respect," and culturally safe care includes building trust, recognizing the role of socioeconomic conditions, communicating respect for patient beliefs and ensuring patients are partners in decisions about their health.

"For providers, cultural competency starts with the understanding that they should not make assumptions about people's culture. Culturally competent staff know to ask questions about what their patients need, find out how to get it, and are willing to advocate for it, such as a request to have a traditional

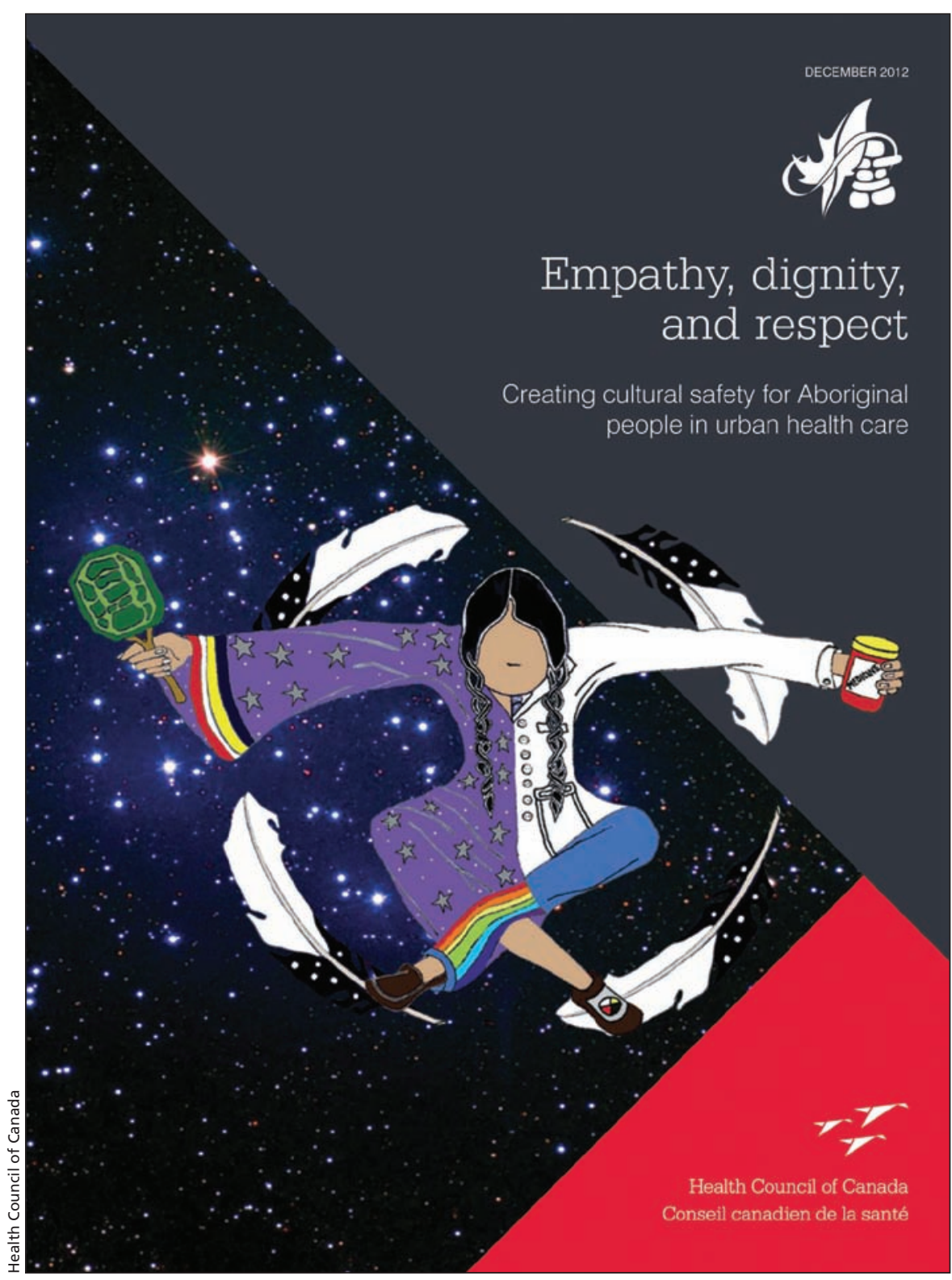

Aboriginal Canadians often feel intimidated and alienated by health care professionals and delay seeking medical care, drop out of treatment early and miss out on the benefits of preventive care, according to a report by the Health Council of Canada.

ceremony or involve a traditional healer in care," the report states. "They also recognize that Aboriginal communities have different beliefs, cultural practices, and languages, and that not all Aboriginal people are interested in following traditional ways."

The health council conducted multiple meetings across the country and found that Aboriginal Canadians including First Nations, Inuit and Métis people - often feel intimidated and alienated by health care professionals. They tend to avoid or delay seeking care, drop out of treatment early and also miss out on the benefits of screening tests, immunizations and other forms of preventive care. 
This should be of particular concern to health care providers, suggests the report, because Aboriginal people are already more likely than other Canadians to have infectious diseases, chronic conditions and die prematurely. The poorer health outcomes in these populations have been linked to poverty, poor nutrition, inadequate housing and obesity, among other factors.

Mainstream health care providers often assume Aboriginal people have the same needs and perspectives as other patients, says Abbott, and this view will likely persist unless they are educated about cultural differences. Fortunately, some have already begun that process.
"We were glad to hear that many of the regions and urban centres are really starting to address these issues by putting in programs and services that reflect the needs of the providers as well as the populations they're serving," says Abbott.

British Columbia, for example, launched the online Indigenous Cultural Competency Training Program in 2010, which provides "fundamental information and encourages self-reflection" about various aspects of Aboriginal culture. Manitoba and Newfoundland and Labrador both have Aboriginal "patient navigators" who provide support and act as a bridge between health care providers and Aboriginal patients.
Saskatchewan has an Aboriginal Home Care Project.

"We've identified in the report over 100 programs, services or organizations that are actively dealing with cultural competency or cultural safety in the delivery of health care services across the country," says Abbott.

Still, there are many regions where cultural competency and cultural safety remain foreign concepts, suggests Abbott, so more change is needed. "There has to be a cultural shift in the attitudes and behaviour of our health care providers." - Bonnie O'Sullivan, CMAJ

CMAJ 2013. DOI:10.1503/cmaj.109-4376 